\title{
Concomitant hiatal hernia repair during bariatric surgery: does the reinforcement make the difference?
}

\author{
Cristian E. BORU *, Pietro TERMINE, Pavlos ANTYPAS, \\ Angelo IOSSA, Maria C. CICCIORICCIO, Francesco DE ANGELIS, \\ Alessandra MICALIZZI, Gianfranco SILECCHIA
}

Division of General Surgery \& Bariatric Center of Excellence-IFSO EC, Department of Medico-Surgical Sciences and Biotechnologies, Sapienza University, Rome, Italy

*Corresponding author: Cristian E. Boru, Division of General Surgery \& Bariatric Center of Excellence-IFSO EC, Department of Medico-Surgical Sciences and Biotechnologies, Sapienza University, Rome, Italy. E-mail: drcrisb@gmail.com

\section{A B S T R A C T}

BACKGROUND: Hiatal hernia repair (HHR) is still controversial during bariatric procedures, especially in case of laparoscopic sleeve gastrectomy (LSG). Aims: to report the long-term results of concomitant HHR, evaluating the safety and efficacy of posterior cruroplasty (PC), simple or reinforced with biosynthetic, absorbable Bio- $\mathrm{A}^{\circledR}$ mesh (Gore, Flagstaff, AZ, USA). Primary endpoint: PC's failure, defined as symptomatic HH recurrence, nonresponding to medical treatment and requiring revisional surgery.

METHODS: The prospective database of 1876 bariatric operations performed in a center of excellence between 20112019 was searched for concomitant HHR. Intraoperative measurement of the hiatal surface area (HSA) was performed routinely.

RESULTS: A total of 250 patients undergone bariatric surgery and concomitant HHR (13\%). Simple PC (group A, 151 patients) was performed during $130 \mathrm{LSG}, 5$ re-sleeves and 16 gastric bypasses; mean BMI $43.4 \pm 5.8 \mathrm{~kg} / \mathrm{m}^{2}$, HSA mean size $3.4 \pm 2 \mathrm{~cm}^{2}$. Reinforced PC (group B) was performed in 99 cases: 62 primary LSG, 22 LGB and 15 revisions of LSG; mean BMI $44.6 \pm 7.7 \mathrm{~kg} / \mathrm{m} 2$, HSA mean size $6.7 \pm 2 \mathrm{~cm}^{2}$. PC's failure, with intrathoracic migration (ITM) of the LSG was encountered in 12 cases $(8 \%)$ of simple vs. only 4 cases $(4 \%)$ of reinforced $\mathrm{PC}(\mathrm{P}=0.23)$; hence, a repeat, reinforced $\mathrm{PC}$ and R-en-Y gastric bypass (LRYGB) was performed laparoscopically in all cases. No mesh-related complications were registered perioperatively or after long-term follow-up (mean 50 months). One case of cardiac metaplasia without goblet cells was detected 4 years postoperatively; conversion to LRYGB, with reinforced redo of the PC was performed. The Cox hazard analysis showed that the use of more than four stitches for cruroplasty represents a negative factor on recurrence $(\mathrm{HR}=8 ; \mathrm{P}<0.05)$.

CONCLUSIONS: An aggressive search for and repair of $\mathrm{HH}$ during any bariatric procedure seems advisable, allowing a low HH recurrence rates. Additional measures, like mesh reinforcement of crural closure with biosynthetic, absorbable mesh, seem to improve results on long term follow-up, especially in case of larger hiatal defects. In our experience, reinforcement of even smaller defects seems advisable in obese population.

(Cite this article as: Boru CE, Termine P, Antypas P, Iossa A, Ciccioriccio MC, de Angelis F, et al. Concomitant hiatal hernia repair during bariatric surgery: does the reinforcement make the difference? Minerva Chir 2020;75:000-000. DOI: 10.23736/ S0026-4733.20.08503-X)

KEYWORDS: HERNIA, hiatal; Bariatric surgery; Laparoscopy.

$\mathrm{I}$ ncidence of symptomatic hiatal hernia $(\mathrm{HH})$ ranges between $10-20 \%$ in the general, adult population. While its implications and indica- tions for repair differ between the different types of $\mathrm{HH}$, the common incidence of gastroesophageal reflux disease (GERD) advocates for anti- 
reflux surgery. ${ }^{1}$ The incompetence of the antireflux barriers and of the crural diaphragm ensues a vicious cycle of symptoms that accentuates and maintains GERD. ${ }^{2}$ Obesity is an independent risk factor for $\mathrm{HH}$ and GERD. ${ }^{3-5}$ Increase of body mass index (BMI) will upsurge the risk of HH's incidence, GERD symptoms and erosive esophagitis (EE), regardless of demographic features and dietary intake. ${ }^{2}$ The prevalence of GERD in patients with obesity ranges from 37\% to $72 \%$, especially in morbid obese patients' candidate to bariatric surgery. ${ }^{2-5}$

Concomitant HHR during bariatric surgery is gaining worldwide popularity, ${ }^{4}$ following international guidelines of SAGES recommending the repair of all hiatus defects during any bariatric procedure, ${ }^{5}$ especially during laparoscopic sleeve gastrectomy (LSG). LSG has higher reported GERD rates and proton pump inhibitor (PPI) use; other indications for concomitant treatment are prevention of postoperative acid/ biliary gastroesophageal reflux and of the intrathoracic migrations (ITM) of the operated stomach, even in asymptomatic patients, especially with large HH. Even if concomitant HHR during LSG, particularly in patients with GERD, is safe in the long term without higher mortality risk 6,7 this issue still remains controversial. Additionally, there is no consensus concerning the preferred surgical option in patients with large $\mathrm{HH}$ and no GERD symptoms or endoscopic evidence for reflux. ${ }^{8}$
Crura's closure, in case of a widened diaphragmatic hiatus, is performed with simple interrupted sutures in primary posterior cruroplasty (PC). However, inadequate PC or postoperative disruption of sutures with subsequent intrathoracic migration was observed to be the primary cause of anatomical failure in HHR, HH recurrence and revisional surgeries. ${ }^{9}$ This led to the technique, among many others, of reinforcing the hiatal closure with prosthetic mesh ${ }^{10}$ in order to decrease recurrence; there are conflictive opinions on mesh use, composition, shape, fixation techniques and associated long-term complications. There is also no consensus on the exact hernia size that should be repaired with a mesh as the definition of hernia size varies across studies. Pre- or intraoperative assessment of the hiatal surface area (HSA) was recently proposed, ${ }^{11}$ due to a positive association between increasing HSA, increasing recurrence risk and therefore a higher need for mesh. A simplified, already established calculation of the HSA as a rhombus area was introduced, measuring the major horizontal distance between the two crura (d1) and the vertical distance between the pillars' decussation and the hiatus' superior edge (d2); $\mathrm{HSA}=\mathrm{d} 1 \mathrm{Xd} 2 / 2^{12}$ (Figure 1 ).

Moreover, the lack of long-term follow up delineating the effectiveness of mesh reinforcement is compounded by the fact that in addition to surgical techniques, factors contributing to the
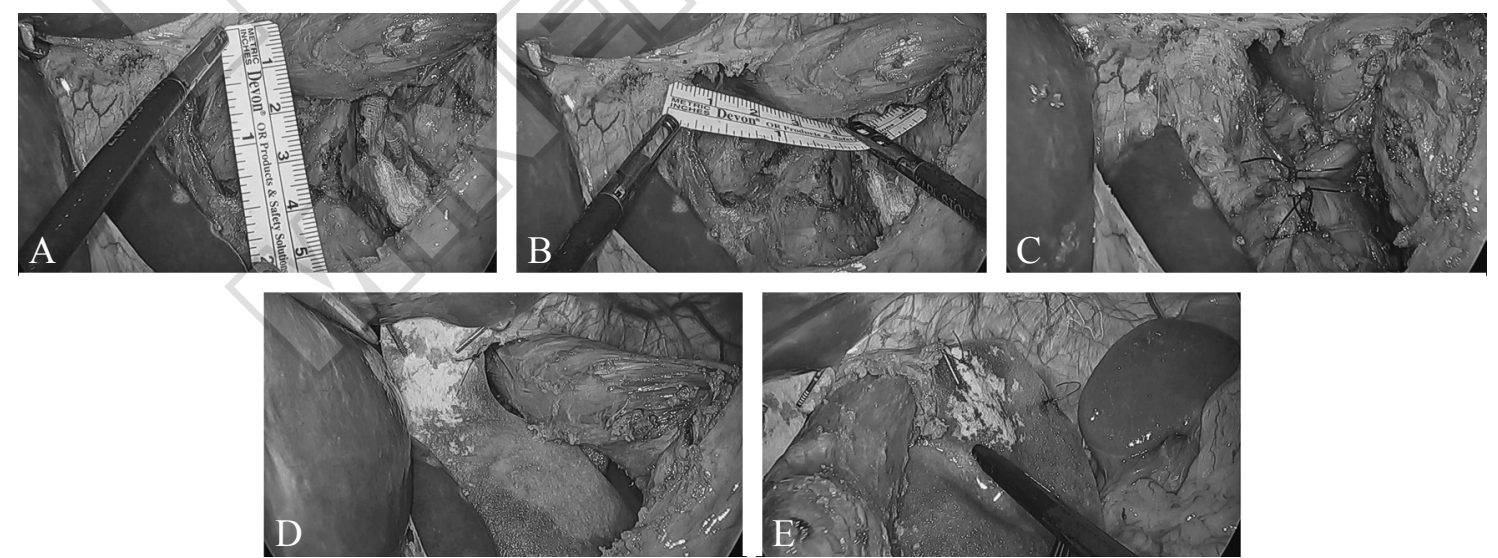

Figure 1.- Intraoperative photo of hiatal defects measurement, posterior cruroplasty and reinforcement with biosynthetic, absorbable Bio-A ${ }^{\circledR}$ mesh (Gore, USA): A) the major horizontal distance between the two crura (d1); B) the vertical distance between the pillars' decussation and the hiatus' superior edge (d2); C) posterior cruroplasty calibrated on $18 \mathrm{Ch}$ bougie; D,E) reinforcement of the posterior cruroplasty with absorbable mesh fixated with absorbable stitches and glue. 
etiology of $\mathrm{HH}$, such as ultrastructural changes of the crura ${ }^{12}$ or pharyngoesophageal ligament can lead to hiatoplasty disruption. ${ }^{13}$

Mesh available for HHR are non-absorbable synthetic, biological and biosynthetic absorbable. Non-absorbable synthetic have a major concern due to related complications, like erosions, local infections, dysphagia, fibrosis, stenosis of the esophago-gastric junction; ${ }^{14,} 15$ the biological, absorbable scaffolds made from decellularized human, bovine or porcine tissues rich in collagen, but their high cost, difficulty in handling and fixation and the possibility of immunological reactions are also reasons for concern. ${ }^{15}$ The absorbable biosynthetic scaffolds are hybrid meshes that exploit the advantages, while minimizing the disadvantages of synthetic and biological prosthesis. Gore ${ }^{\circledR}$ Bio- $A^{\circledR}$ Tissue Reinforcement (Gore, Flagstaff, AZ, USA)is currently the only biosynthetic, absorbable mesh with CE mark (since 2008) and Food and Drug Administration's (FDA, USA) approval (since 2017) for hiatal use, showing good technical results, ${ }^{16}$ with no local or general complications and apparently efficient in reducing postoperative recurrence on medium and long-term.6, 7, 17 Bio- $\mathrm{A}^{\circledR}$ is composed of a 3 -dimensional web of copolymer of polyglycolic acid (67\%) and trimethylene carbonate $(33 \%)$, providing a temporary scaffold gradually absorbed over 6 months and replaced by vascularized soft tissue, leaving no permanent material behind. 18,19

The aim of this study was to report the longterm results of a single institution series of HHR during bariatric surgery, evaluating PC's safety and efficacy, simple or reinforced with a biosynthetic, absorbable mesh. The primary endpoint of this study was the rate of PC's failure. Secondary endpoints were postoperative complications related to $\mathrm{PC}$, dysphagia rate, GERD symptoms control (in terms of PPI therapy needing), endoscopic findings, and re-operation rate.

\section{Materials and methods}

This is a retrospective study on a prospective database of 1876 morbid obese patients that underwent bariatric surgery in our Center of Excellence between 2011-2019; most commonly laparoscopic procedures performed were LSG (1485, 79\%), standard R-en-Y gastric bypass LRYGB (176, 9.3\%), one anastomosis gastric bypass LOAGB/MGB (131, 7\%), both as primary and revisional surgery. Other procedures included: gastric banding and gastric plication, both abandoned from 2012; duodenal switch BPD-DS, single anastomosis duodeno-ileal bypass with sleeve SADI-S, revisions of the LSG, revisional bariatric procedures. Patients that underwent concomitant HHR simple (group A) or reinforced (group B) during any bariatric surgery were included in this study, designed and approved by the local board.

Preoperative workup and surgical technique have been illustrated in detail elsewhere. ${ }^{20}$ All patients' candidate to bariatric surgery underwent preoperatively esophagogastroduodenoscopy (EGDS) with helicobacter pylori (HP) testing. Contrast X-ray studies with barium or CT scan with $3 \mathrm{D}$ reconstruction were indicated in case of endoscopic diagnosis of large HH. All symptomatic GERD patients received preoperatively PPI. Patients demographics, obesity-related comorbidities, PPI use, mortality, conversion rate, operative time, intra- and perioperative (30 days) complications Clavien-Dindo classification, ${ }^{21}$ postoperative hospital stay, mesh-related complications, resolution or persistence of GERD symptoms, endoscopic esophageal lesions and clinical and/or radiological $\mathrm{HH}$ recurrences, were prospectively evaluated and recorded in a devoted database. Exclusion criteria were BMI $\left(>60 \mathrm{~kg} / \mathrm{m}^{2}\right)$ and type IV HH.

Briefly, the surgical technique of concomitant HHR during laparoscopic bariatric surgery was usually performed before the particular bariatric technique and consisted in full exposure of the hiatal area, HH reduction, abdominalization of the esophagus for at least $3 \mathrm{~cm}$ and HSA intraoperative measurement. ${ }^{11,22}$ Patients with sliding $\mathrm{HH}$ and HSA $<4 \mathrm{~cm}^{2}$ underwent simple PC with interrupted nonabsorbable sutures (Prolene ${ }^{\circledR}$ polypropylene 1/0, Ethicon Endosurgery, New Brunswick, NJ, USA), while patients with larger hiatal defect $\left(>4 \mathrm{~cm}^{2}\right)$ and pillars' weakness underwent on-lay mesh-reinforced PC (Figure 1A-C). Mesh was tailored to $8 / 7 \mathrm{~cm}$, cutting the left part of the mesh, creating a hole in the up- 


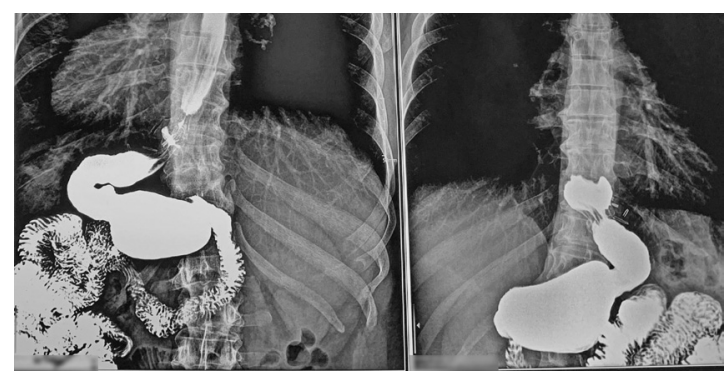

Figure 2.--Radiological, asymptomatic hiatal hernia recurrence $(<2 \mathrm{~cm})$ seen on contrast X-ray study 12 months postoperative after LSG and concomitant, simple posterior cruroplasty for initially symptomatic hiatal hernia. EGDS negative for esophageal lesions and patient does not need PPI therapy.

per part for the esophagus passage, obtaining an asymmetrical " $u$," with the right part longer for adequate right pillar reinforcement. Afterwards, the mesh, rolled like a cigarette, was inserted through the 10-mm trocar, superimposed on the PC and fixed with absorbable $2 / 0$ stitches and glue application (Figure 1D, E).

Postoperative HHR check-up consisted in upper gastrointestinal contrast X-ray studies every 12 months, with any hiatal hernia seen on the radiological contrast image $<2 \mathrm{~cm}$ in length defined as "radiological asymptomatic recurrence" (Figure 2).22 EGDS surveillance was recommended routinely every 24 months or whenever symptomatology or radiological examination required. Barium swallow and CT-scan with 3D reconstruction was indicated when EGD showed recurrent $\mathrm{HH}$, to demonstrate the ITM. Esophageal 24hour $\mathrm{pH} /$ impedance reflux monitoring completed the work-up in selected cases, in order to measure the amount of gastro-esophageal reflux (both acidic and non-acidic) and to assess whether the symptoms were correlated with the reflux.

Other criteria used was the obstinacy of PPI therapy, which is routinely interrupted 6 months after initial bariatric procedure and the continuance of the therapy was considered a persistence or recurrence of the preoperative GERD. The failure of the cruroplasty was defined as recurrence of the $\mathrm{HH}$, defined as disruption or enlargement of the suture repair (see Figure 3, demonstrated by ITM of the operated stomach $\geq 2 \mathrm{~cm}$ (Figure 4), symptomatic for GERD (recurrence, persistence or de novo) and nonresponsive to medical treatment, requiring revisional surgery.

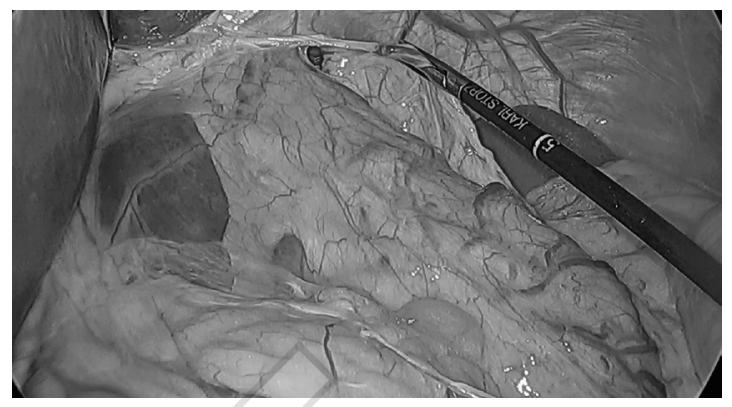

Figure 3.--Intraoperative findings in GERD recurrent, symptomatic patient, of failure of the initial posterior cruroplasty for hiatal hernia repair during LSG, with recurrence of the hiatal hernia, intrathoracic sleeve migration $(>2 \mathrm{~cm})$ and disruption of the surgical suture.
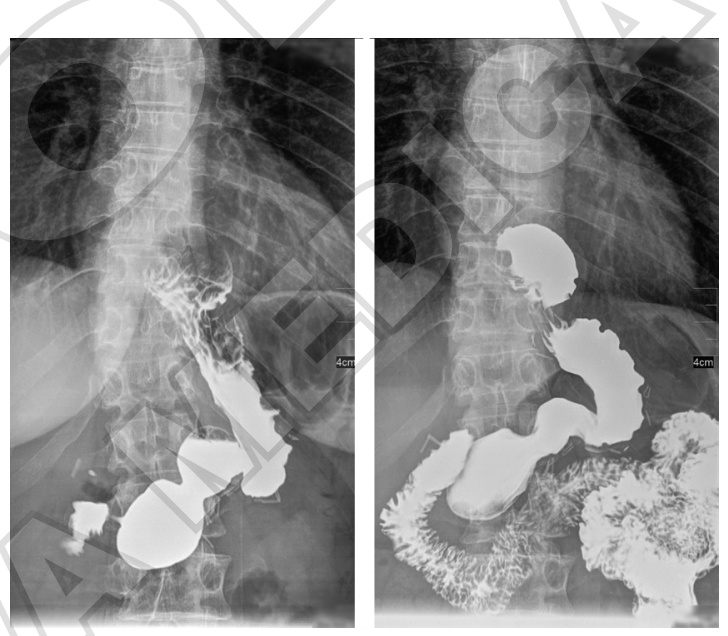

Figure 4.--Intrathoracic sleeve migration $(>2 \mathrm{~cm})$ seen on postoperative contrast X-ray studies with barium, in GERD recurrent, symptomatic patient with previously LSG and simple posterior cruroplasty, defined as failure of the cruroplasty.

Our intention-to-treat consisted in 2 situations: first was the symptomatic failure of the PC, non-responsive to medical treatment; second consisted in GERD recurrence or persistent despite medical treatment, without any evidence of $\mathrm{HH}$ recurrence (no failure of the PC).

\section{Results}

A total of 250 patients undergone bariatric surgery and concomitant HHR (13\%); demographics and types of operation are reported in Table I, II and results in Table III. Simple PC (group A) was performed during $130 \mathrm{LSG}, 5$ re-sleeves and 15 LGB and one LMGB; mean BMI was 
TABLE I.-Demographics, pre- and intraoperative data in 250 patients undergoing bariatric surgery and concomitant hiatal hernia (HH) repair, with simple posterior cruroplasty $(P C)$ in group A vs. reinforced $P C$ with Bio- $A$ biosynthetic, absorbable mesh in group B. Preoperative endoscopic findings (performed in all patients included in the study).

\begin{tabular}{lccc}
\hline Demographics & Group A $(151 \mathrm{pts})$ simple PC & Group B $(99$ pts) reinforced PC & P value \\
\hline Age (years Mean \pm SD) & $46.6 \pm 10.1$ & $47.5 \pm 12.1$ & 0.67 \\
Sex $(\mathrm{M} / \mathrm{F})$ & $35 / 116$ & $22 / 77$ & 0.86 \\
BMI $\left(\mathrm{kg} / \mathrm{m}^{2}\right.$, Mean $\left.\pm \mathrm{SD}\right)$ & $43.4 \pm 5,8$ & $44.6 \pm 7.7$ & 0.13 \\
Primary/revisional surgery & $138 / 13$ & $83 / 16$ & 0.070 \\
Symptomatic patients & 52 & 44 & 0.0001 \\
Preoperative HH's diagnosis & $102(67.5 \%)$ & $72(72.7 \%)$ & 0.38 \\
Intraoperative HH's diagnosis & $49(32.4 \%)$ & $27(27.7 \%)$ & 0.38 \\
Endoscopy and biopsy & & $53(53.5 \%)$ & 0.0001 \\
GERD symptoms & $45(29.8 \%)$ & $25(25.2 \%)$ & 0.0006 \\
Esophagitis & $14(9.2 \%)$ & $48 / 53(90.5 \%)$ & 0.61 \\
Responder to preoperative PPI therapy & $42 / 45(93.3 \%)$ & $28(28.2 \%)$ & 0.15 \\
NERD & $31(20.5 \%)$ & 0 & 0.68 \\
Barrett preoperative & 1 & & 7 \\
\hline
\end{tabular}

TABLE II.-Laparoscopic bariatric procedures performed in concomitancy with posterior cruroplasty (PC) during 2011-2019 in a Bariatric Center of Excellence.

\begin{tabular}{lcc}
\hline Bariatric procedures with concomitant PC & Group A (151 pts) simple PC & Group B (99 pts) reinforced PC \\
\hline LSG & 130 & 62 \\
Re-sleeves & 5 & 5 \\
PC after LSG & 0 & 10 \\
Primary LRYGBP & 15 & 22 \\
Primary LOAGB/MGB & 1 & 0 \\
Total patients & 151 & 99 \\
\hline
\end{tabular}

LSG: sleeve gastrectomy; LRYGBP: R-en-Y gastric bypass; LOAGB/MGB: single anastomosis gastric bypass.

TABLE III.-Results after concomitant posterior cruroplaty PC during bariatric surgery, simple (group A) vs. reinforced (group B). Barrett cardiac metaplasia without goblet cells. Postoperative esophagitis registered in group A ( 8 class $A, 5$ class $B$ and 2 class $C$ ) and in group B (5 class $A, 1$ class $B$ and 2 class $C)$.

\begin{tabular}{lccc}
\hline Results & Group A simple PC & Group B reinforced PC & P value \\
\hline Mortality rate & 1 & 0 & 0.68 \\
Conversion to open & 0 & 1 & 0.35 \\
Mean operative time (minutes) & $105 \pm 38.2$ & $141 \pm 52.2$ & 0.0001 \\
Postoperative stay (days) & $3 \pm 1.29$ & $4 \pm 1.43$ & 0.0003 \\
Mean follow-up (range) & 49.1 months & 50.9 months & 0.023 \\
Drop-out (patients) & $11(7.3 \%)$ & $5(5 \%)$ & 0.48 \\
GERD symptoms persistent postoperatively & $15(33.3 \%)$ & $14(26.4 \%)$ & 0.45 \\
De novo GERD & $15(10.7 \%)$ & $12(12.7 \%)$ & 0.63 \\
PPI therapy after surgery & $30(21.4 \%)$ & $26(27.6 \%)$ & 0.27 \\
Postoperative Esophagitis & $15(21.1 \%)$ & $8(14.5 \%) ;$ & 0.34 \\
Barrett postoperative & 0 & 1 & 0.35 \\
Transient dysphagia $<6$ weeks & $15(10 \%)$ & $8(\%)$ & 0.62 \\
Mesh-related complications & 0 & 0 & - \\
\hline
\end{tabular}

$43.3 \pm 5.8 \mathrm{~kg} / \mathrm{m}^{2}$ and HSA mean size was $3.4 \pm 2$ $\mathrm{cm}^{2}$. Reinforced PC (group B) was performed in 99 cases: 62 primary LSG, 22 GB and 15 different revisions of LSG; mean BMI $44.6 \pm 7.7 \mathrm{~kg} / \mathrm{m}^{2}$ and HSA mean size $6.7 \pm 2 \mathrm{~cm}^{2}$.

In a total of 174 patients $(69.6 \%)$, the $\mathrm{HH}$ di- agnosis was made preoperative: in $102(67.5 \%)$ patients of group A and $72(72.7 \%)$ of group $\mathrm{B}$, without any significant difference $(\mathrm{P}>0.3)$. Eighty patients were asymptomatic despite the preoperative $\mathrm{HH}$ diagnosis (50 in group A, 28 in group B). A statistically significant higher 
number of GERD symptomatic patients were encountered preoperatively in group B 53.5\% vs $29.8 \%$ in group $\mathrm{A}, \mathrm{P}=0.0001$. The endoscopic findings are reported in Table I, showing a higher percentage of esophagitis in group B $25.2 \%$, $\mathrm{P}<0.0006$ : Los Angeles classification (LAC) A in 11 patients, 9 with class $B$ and 5 with class $C$ $(\mathrm{P}=0.0006)$, while in group $\mathrm{A}$ consisted in LAC A in 11 patients, 3 with class B. Class B or C was a certain contraindication to LSG. One case of cardiac metaplasia was detected preoperatively in a patient with symptomatic HH; a LRYGB and simple PC was performed, and endoscopic control after 24 and 48 months showed complete resolution. NERD was detected in $31(20.5 \%)$ patients of group A and in 28 (28.2\%) of group $\mathrm{B}$, without significant difference between them. All symptomatic patients were in preoperative PPI therapy, continuously in $77 \%$ and on demand in $23 \%$. The PPI response rate in symptomatic GERD patients was high in both groups (group A $93.3 \%$ vs group B $90.5 \%$; P $>0.6$ ). The median preoperative GERD-HRQL score of all the patients was 16 .

Mean operative time was $121 \pm 57.05 \mathrm{~min}$ for all patients, and it was significantly longer in B group ( $141 \pm 52.3 \mathrm{~min}, \mathrm{P}=0.0001)$. The conversion rate to open surgery was $0.4 \%$ ( 1 case of intraoperative onset of ventricular fibrillation requiring defibrillation in group B). Mortality rate was 0.4 (1 case of leak in group A, complicated with ITM and mediastinitis in the early postoperative course). Mean postoperative stay was $3.8 \pm 1.38$ days; it was statistically significant longer for group B $(4 \pm 1.43$ days, $\mathrm{P}=0.05)$. Perioperative complications occurred in 13 patients out of $250(5.2 \%)$; 6 patients (4\%) in group $\mathrm{A}$ and $7(7 \%)$ in group B. In group A, complications were as follow: grade II ( 2 cases), grade IIIa (2 cases) and grade IIIb (one case): two bleedings, one pleural effusion, one midgastric stenosis and one incisional hernia; grade V 1 death due to leak complicated by mediastinitis. In group B, the perioperative complications were grade I (1 case of fever and vomiting); grade II (3 bleedings and one pleural effusion), grade IIIa: one bleeding treated with percutaneous drainage and one gastric leak successfully treated with selfexpandable metal stent.
Mean follow-up was 50 months, 49.1 in group A and 50.9 in group B, respectively. Drop-out rate was excellent: 11 patients in group $\mathrm{A}(7.3 \%)$, from which 2 deaths for independent causes and 5 patients $(5 \%)$ in group B.

A total of 45 (29.8\%) patients of group A and $53(53.5 \%)$ patients of group B reported GERD symptoms preoperatively. During the postprocedural follow-up, 15/45 (33.3\%) cases in group A and $14 / 53(26.4 \%)$ cases in group B declared persistent or recurrent GERD ( $\mathrm{P}>0.45)$. Interestingly, significantly less patients of group B suffered by GERD symptoms postoperatively than those of group A $(\mathrm{P}<0.0003)$. Analogically, the use of PPI was not different between the groups postoperatively (group A $21.4 \%$ vs group B 27.6\%; $\mathrm{P}>0.27$ ) but the group B patients showed a significant decrease in PPI therapy after the surgery $(\mathrm{P}<0.0003)$. Control EGDS was performed in 71 $(50.7 \%)$ patients of group A and $55(58.5 \%)$ patients of group B ( $>0.2)$. Symptomatic patients were $30(21.4 \%)$ in group A and $26(27.6 \%)$ in group B, all accepted EGDS control. Endoscopic report of esophagitis was recorded in 15 (21.1\%) cases in group A (8 class A; 5 class B; 2 class $\mathrm{C}$ LAC) and in $8(14.5 \%)$ cases in group B (5 class A; 1 class B; 2 class C LAC) $(\mathrm{P}>0.3)$. The median postoperative GERD-HRQL score of all the patients was 4 .

Failure of the PC as reason of reintervention, with symptomatic intrathoracic migration (ITM) and nonresponsive to medical treatment, was encountered in 12 cases $(8 \%)$ of group A vs. only 4 cases $(4 \%)$ of group $\mathrm{B}(\mathrm{P}=0.23)$ (Table IV); it was assessed by EGDS, contrast X-ray studies and 3D-CT scan). Patients with clinical and radiological recurrence were offered reinforced PC and LRYGB. All cases showed symptom remission and suspended PPI regimen within 3 months after surgery. Only one case of HHR's failure was encountered in the LRYGB group, surgically reviewed as well (anterior cruroplasty).

One case of cardiac metaplasia without goblet cells (Figure 5) ${ }^{23}$ was detected in a patient with LSG and failed reinforced PC (7 cm ITM of the LSG after 4 years), successfully converted to LRYGB, with $\mathrm{HH}$ reduction and reinforced redo of the PC; at 18 months of follow-up, no Barrett's recurrence was recorded. The Cox hazard 
TABLE IV.-Reasons to conversion LRYGB: failure of the posterior cruroplasty PC was defined as symptomatic hiatal hernia's recurrence, with intrathoracic migration $>2 \mathrm{~cm}$, nonresponding to medical treatment and requiring revisional surgery.

\begin{tabular}{lccc}
\hline Reasons to conversion for failure of the PC & $\begin{array}{c}\text { Group A (pts no 151) } \\
\text { simple PC }\end{array}$ & $\begin{array}{c}\text { Group B (pts no 99) } \\
\text { reinforced PC }\end{array}$ & P value \\
\hline Recurrent GERD + failure & $12(8 \%)$ & $4(4 \%)$ & 0.23 \\
Recurrent GERD (no failure) & $2(1.3 \%)$ & $1(1 \%)$ & 0.82 \\
Endoscopic mucosal lesions post-sleeve (metaplasia) & 0 & 1 & 0.93 \\
Overall conversions & 14 & 5 & 0.22 \\
\hline
\end{tabular}

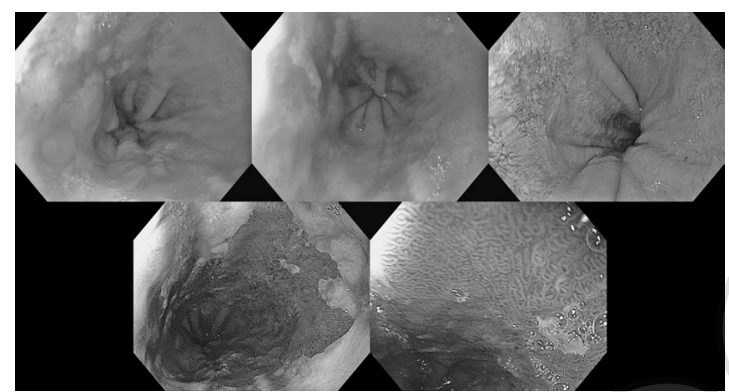

Figure 5.-Endoscopic findings in one case of postoperative Barrett esophagus C1M5 Praga classification ${ }^{23}$ with NBI evaluation (Narrow Band Imaging) after failure of posterior cruroplasty during initial LSG. The patient was submitted to conversion to LRYGB and reinforced posterior cruroplasty.

analysis showed that the use of more than four stitches for cruroplasty represents a negative factor on recurrence $(\mathrm{HR}=8 ; \mathrm{P}<0.05)$.

GERD as reason for reoperation without a failure of the PC was encountered in 3 cases: 2 recurrent cases in group A and one de novo GERD in group B. Local situation was checked before (contrast X-ray studies, EGDS that showed non erosive reflux disease) and during the reoperation, but no revision of the PC was needed; conversion to LRYGBP was performed and all showed symptom remission and suspended PPI within 3 months after surgery.

No case of mesh infections was registered at any time, despite the presence of one case of leak after LSG. No cases of esophageal stenosis or erosion were registered during the EGDS performed postoperatively. Additionally, no cases of postoperative hiatal area's fibrosis/distortion were recorded by $\mathrm{CT}$ scan performed for recurrent cases or in cases needing surgical revision $(\mathrm{N} .=4)$. Only transient dysphagia was reported in 12 patients: 15 in group A (10.4\%) and 8 in group B (8\%) and resolved within 6 weeks by diet counseling and prokinetic drugs (Table III).

\section{Discussion}

Concomitant HHR is already an established reality during bariatric surgery.6, 24, 25 Recent studies accomplished that HHR in patients undergoing LSG reduces GERD rates after surgery. The first systematic review to examine the efficacy and technical aspects of simultaneous LSG and HHR concluded that the combined approach is safe and effective and results in acceptable postoperative GERD rates, with 16 of 17 studies showing good outcomes; the authors therefore recommended this combined approach. ${ }^{24}$ Eighteen \% of the patients operated by North American centers, participating to the Metabolic and Bariatric Surgery Accreditation and Quality Improvement Program (MBSAQIP), underwent concurrent HHR during LSG. ${ }^{25}$ From 130.000 patients participating to the same program, concomitant HHR was significantly more common during LSG compared to LRYGB $(21.0 \%$ vs $10.8 \%, \mathrm{P}<0.0001$ ), despite the lower preoperative GERD rate. These results not only suggest a marked difference in the intraoperative management of $\mathrm{HH}$ during bariatric surgery procedures, but also underline the fact that PC was used in up to $31 \%$ of the surgeries, in order to prevent recurrences and GERD. Therefore, in order to provide good technical results, it is imperative to assume that $\mathrm{HH}$ should be searched and repaired during LSG. ${ }^{8}$ LRYGB patients are more likely to have preoperative GERD compared to LSG and the efficacy and success of LRYGB in reducing GERD symptoms up to 3 years postoperatively has already been demonstrated. ${ }^{25}$ Hence, the lower rate of concurrent HHR in LRYGB patients could be attributed to the overall success rate of LRYGB in treating GERD symptoms, possibly attributed to the minimization of acid production in addition to bile diversion, inherent components of 
this procedure. However, a less aggressive approach to HHR during LRYGB, especially in large hiatal defects, may lead to less than favorable outcomes. In our experience, concomitant HHR during bypass procedures was used in 38 patients, with a higher distribution in the larger hiatal defects group B $\left(>4 \mathrm{~cm}^{2}\right)$; in only one case surgical revision was needed for GERD persistence, when anterior cruroplasty was performed in addition to the initial reinforced PC.

Strategies focused on the development of new type of mesh, like the slowly absorbable biosynthetic meshes, have been introduced recently to combine the advantages of both a synthetic mesh (no degradation shortly after implantation) and a biological mesh (the remodeling aspects and the better tolerance in case of contamination). These advantages, combined with the proven complete absorption 7 months after positioning, 18 made us choose the Bio- $\mathrm{A}^{\circledR}$ mesh for the PC's reinforcement. Our experience with concomitant HHR during bariatric surgery was gradually analyzed and published lately, focusing on the outcomes of long-term follow-up, especially in the reinforced group with Bio-A ${ }^{\circledR}$ mesh. ${ }^{6,17,20,22}$ Surgical technique's choice 22 was made on a multifactorial base: patient's demographics, the dimensions of the hiatal defect (routine intraoperative HSA measurement), score of esophagitis, and severity of symptoms; no long-term complications were recorded due to the concomitant $\mathrm{PC}$, not even in the case when bioabsorbable, synthetic mesh was used (no mesh-related complications, no long-term dysphagia). Second report of our series was on the results of a prospective randomized study comparing concomitant PC during LSG, 48 cases with simple vs 48 cases of reinforced PC and it was updated recently with results after 5 years follow-up. ${ }^{20}$ The general trend confirmed that reinforced cruroplasty was highly effective in a certain subgroup of patients with weakness of the pillars and medium hiatal defect (range 4-8 $\mathrm{cm}^{2}$ ), with no side effects or complications related to the use of the totally absorbable synthetic mesh. Even so, patients should be informed about a possible recurrence. At the same time, an acceptable rate (80\%) of GERD symptoms control was achieved on long-term followup. Successively, results on 92 consecutive pa- tients with reinforced PC during bariatric surgery with mid-term follow-up (mean 41 months) supported the use of absorbable mesh for HHR (safe profile with nil complications rate), showing excellent recurrence rate results $(5.4 \%)$ and good GERD symptoms control. ${ }^{17}$ The present analysis has completed the results of the overall experience in 250 consecutive patients, confirming the role of PC in the prevention of $\mathrm{HH}$ recurrence. This was seen especially in the reinforced group ( $4 \%$ failure vs. $8 \%$ in the group with simple PC), even if patients with a larger HSA (anatomical difference) and higher incidence of GERD were included in this group.

When speaking strictly about LSG and concomitant PC, we report a combined $\mathrm{HH}$ recurrence in 15 cases with ITM out of 192 cases (7.8\%), which is an underestimated complication of LSG, commonly seen in patients with central obesity, chronic constipation, and post-LSG GERD. ${ }^{26}$ Due to the higher incidence at long term of cruroplasty's failure in group A (simple PC) in respect to group B (reinforced PC), it seems reasonable to recommend in the obese population the cruroplasty's reinforcement, but this would be amended by costs, availability and technical skills. The combined cruroplasty's failure rate $(6.4 \%)$ at 5 years after bariatric surgery and concomitant PC is similar or less than studies on traditional antireflux surgery. ${ }^{27}$

Limitations of the study are determined by the incomplete follow-up (approximatively $10 \%$ of patients are lost to follow-up) and the incomplete endoscopic postoperative surveillance. EGDS was considered an invasive investigation; only $51.6 \%$ of the asymptomatic patients accepted it. All symptomatic GERD patients underwent postoperative EGDS, and severe esophagitis B or C was found in 11 patients, with only one case of Barrett's esophagus identified that were consecutively converted to LRYGBP. This is in contradiction with some recent studies that showed an increase of Barrett's esophagus after LSG on long-term follow- up, even in asymptomatic patients. ${ }^{8}$ So, our findings related to the cruroplasty's failure (a combined rate of $\mathrm{HH}$ recurrence at 4 years of $6.4 \%$ ) are driven by the symptomatology accused by the patients that returned to us, seeking for treat- 
ment. We don't know the exact percentage of failure in the whole study group, because asymptomatic patients might not be investigated completely for recurrence and refused EGDS. We consider another limitation determined by the endoscopic Hill classification of $\mathrm{HH}$ only recently introduced in our practice, ${ }^{28}$ that was shown to be slightly stronger compared to the axial length of a $\mathrm{HH}$ and might replace in the general practice the axial length in the endoscopic assessment of the mechanical antireflux barrier of the gastroesophageal junction. Nevertheless, we introduced in the last 2 years the expressed, compulsory endoscopic surveillance of LSG patients every 2 years. Other limitation might derive by the partially controlled use of PPI postoperative, which was overused in some patients, due to the availability of easy prescriptions from the general practitioner.

\section{Conclusions}

An aggressive search for and repair of $\mathrm{HH}$ during any bariatric procedure seems advisable, allowing a low $\mathrm{HH}$ recurrence rates. Additional measures, like mesh reinforcement of crural closure with biosynthetic, absorbable mesh, seem to improve results on long term follow-up, especially in case of larger hiatal defects. In our experience, reinforcement of even smaller defects seems advisable in obese population.

\section{References}

1. Sfara A, Dumitrascu DL. The management of hiatal hernia: an update on diagnosis and treatment. Med Pharm Rep 2019;92:321-5.

2. Mittal RK, Zifan A, Kumar D, Ledgerwood-Lee M, Ruppert E, Ghahremani G. Functional morphology of the lower esophageal sphincter and crural diaphragm determined by three-dimensional high-resolution esophago-gastric junction pressure profile and CT imaging. Am J Physiol Gastrointest Liver Physiol 2017;313:G212-9.

3. Oor JE, Roks DJ, Ünlü Ç, Hazebroek EJ. Laparoscopic sleeve gastrectomy and gastroesophageal reflux disease: a systematic review and meta-analysis. Am J Surg 2016;211:250 67.

4. Docimo S Jr, Rahmana U, Bates A, Talamini M, Pryor A, Spaniolas K. Concomitant hiatal hernia repair is more common in laparoscopic sleeve gastrectomy than during laparoscopic Roux-en-Y gastric bypass: an analysis of 130,772 cases. Obes Surg 2019;29:744-6.

5. Kohn GP, Price RR, DeMeester SR, Zehetner J, Muensterer OJ, Awad Z, et al.; SAGES Guidelines Committee.
Guidelines for the management of hiatal hernia. Surg Endosc 2013;27:4409-28.

6. Janik MR, Ibikunle C, Aryaie AH. Safety of concurrent sleeve gastrectomy and hiatal hernia repair: a propensity score-matched analysis of the MBSAQIP registry. Surg Obes Relat Dis 2020;16:365-71.

7. Boru CE, Coluzzi MG, de Angelis F, Silecchia G. LongTerm Results After Laparoscopic Sleeve Gastrectomy with Concomitant Posterior Cruroplasty: 5-Year Follow-up. J Gastrointest Surg 2020;24:1962-8.

8. Assalia A, Gagner M, Nedelcu M, Ramos AC, Nocca D. Gastroesophageal Reflux and Laparoscopic Sleeve Gastrectomy: Results of the First International Consensus Conference. Obes Surg 2020;30:3695-705.

9. Granderath FA, Kamolz T, Schweiger UM, Pointner R. Laparoscopic refundoplication with prosthetic hiatal closure for recurrent hiatal hernia after primary failed antireflux surgery. Arch Surg 2003;138:902-7.

10. Sathasivam R, Bussa G, Viswanath Y, Obuobi RB, Gill T, Reddy A, et al. 'Mesh hiatal hernioplasty' versus 'suture cruroplasty' in laparoscopic para-oesophageal hernia surgery; a systematic review and meta-analysis. Asian J Surg 2019;42:53-60.

11. Boru CE, Rengo M, Iossa A, De Angelis F, Massaro M, Spagnoli A, et al. Hiatal Surface Area's CT scan measurement is useful in hiatal hernia's treatment of bariatric patients [published online ahead of print, 2019 Oct 31]. Minim Invasive Ther Allied Technol 2019; $\cdots: 1-8$.

12. Fei L, del Genio G, Rossetti G, Sampaolo S, Moccia F, Trapani $\mathrm{V}$, et al. Hiatal hernia recurrence: surgical complication or disease? Electron microscope findings of the diaphragmatic pillars. J Gastrointest Surg 2009;13:459-64.

13. von Diemen V, Trindade EN, Trindade MR. Hiatal hernia and gastroesophageal reflux: study of collagen in the phrenoesophageal ligament. Surg Endosc 2016;30:5091-8.

14. Huddy JR, Markar SR, Ni MZ, Morino M, Targarona $\mathrm{EM}$, Zaninotto G, et al. Laparoscopic repair of hiatus hernia: does mesh type influence outcome? A meta-analysis and European survey study. Surg Endosc 2016;30:5209-21.

15. Elango S, Perumalsamy S, Ramachandran K, Vadodaria $\mathrm{K}$. Mesh materials and hernia repair. Biomedicine (Taipei) 2017;7:16

16. Asti E, Sironi A, Bonitta G, Lovece A, Milito P, Bonavina L. Crura augmentation with Bio-A ${ }^{\circledR}$ mesh for laparoscopic repair of hiatal hernia: single-institution experience with 100 consecutive patients. Hernia 2017;21:623-8.

17. Iossa A, Silecchia G. Mid-term safety profile evaluation of Bio-A absorbable synthetic mesh as cruroplasty reinforcement. Surg Endosc 2019;33:3783-9.

18. Boru CE, Manolescu N, Ulmeanu DI, Copca N, Constantinica V, Copaescu C, et al. Platelet-rich plasma PRP vs. absorbable mesh as cruroplasty reinforcement: a study on an animal model. Minim Invasive Ther Allied Technol 2020;110. [[Epub ahead of print]]

19. Gore WL; Associates. Inc. GORE BIO-A Tissue Reinforcement; 2008 [Internet]. Available from: https://www.goremedical.com/products/bioatissue [cited 2020, Oct 7].

20. Ruscio S, Abdelgawad M, Badiali D, Iorio O, Rizzello M, Cavallaro G, et al. Simple versus reinforced cruroplasty in patients submitted to concomitant laparoscopic sleeve gastrectomy: prospective evaluation in a bariatric center of excellence. Surg Endosc 2016;30:2374-81.

21. Dindo D, Demartines N, Clavien PA. Classification of surgical complications: a new proposal with evaluation in a cohort of 6336 patients and results of a survey. Ann Surg 2004;240:205-13. 
22. Silecchia G, Iossa A, Cavallaro G, Rizzello M, Longo F. Reinforcement of hiatal defect repair with absorbable mesh fixed with non-permanent devices. Minim Invasive Ther Allied Technol 2014;23:302-8.

23. Sharma P, Dent J, Armstrong D, Bergman JJ, Gossner L, Hoshihara Y, et al. The development and validation of an endoscopic grading system for Barrett's esophagus: the Prague C \& M criteria. Gastroenterology 2006;131:1392-9.

24. Mahawar KK, Carr WR, Jennings N, Balupuri S, Small PK. Simultaneous sleeve gastrectomy and hiatus hernia repair: a systematic review. Obes Surg 2015;25:159-66.

25. Madalosso CA, Gurski RR, Callegari-Jacques SM, Navarini D, Mazzini G, Pereira MS. The impact of gastric bypass on gastroesophageal reflux disease in morbidly obese patients. Ann Surg 2016;263:110-6.

26. Saber AA, Shoar S, Khoursheed M. Intra-thoracic sleeve migration (ITSM): an underreported phenomenon after laparoscopic sleeve gastrectomy. Obes Surg 2017;27:1917-23.

27. Armijo PR, Krause C, Xu T, Shostrom V, Oleynikov D. Surgical and clinical outcomes comparison of mesh usage in laparoscopic hiatal hernia repair. Surg Endosc 2020;•••:

28. Hansdotter I, Björ O, Andreasson A, Agreus L, Hellström $\mathrm{P}$, Forsberg A, et al. Hill classification is superior to the axial length of a hiatal hernia for assessment of the mechanical anti-reflux barrier at the gastroesophageal junction. Endosc Int Open 2016;4:E311-7.

Conflicts of interest.-The authors certify that there is no conflict of interest with any financial organization regarding the material discussed in the manuscript.

Authors' contributions.-All authors read and approved the final version of the manuscript.

History.-Article first published online: October 2, 2020. - Manuscript accepted: September 23, 2020. - Manuscript received: July 8,2020 